\title{
Discrete symmetry's chains and links between integrable equations
}

\author{
A.V. Yurov \\ Theoretical Physics Department, \\ Kaliningrad State University, \\ 236041, Al.Nevsky St., 14, Kaliningrad, Russia \\ email yurov@freemail.ru
}

\begin{abstract}
The discrete symmetry's dressing chains of the nonlinear Schrödinger equation (NLS) and Davey-Stewartson equations (DS) are consider. The modified NLS (mNLS) equation and the modified DS (mDS) equations are obtained. The explicitly reversible Bäcklund auto-transformations for the mNLS and $\mathrm{mDS}$ equations are constructed. We demonstrate discrete symmetry's conjugate chains of the KP and DS models. The two-dimensional generalization of the $P_{4}$ equation are obtained.
\end{abstract}

\section{Introduction}

Darboux transformations (DT) are very useful method to construct exact solutions of integrable PDE [1]. Some times ago a new approach to the DT (called dressing chains of discrete symmetries) emerged at the horizon of soliton's mathematics. This approach to proliferate integrable equations was proposed in the example of the Korteweg-de Vries $(\mathrm{KdV})$ and sine-Gordon equations [2]. The scheme to proliferate the integrable equation (we choose the $\mathrm{KdV}$ for definitenes) starts out from $L A$ pair and DT of this equation. After very simple transformations (see Sec. 2) we obtain new integrable equations with their $L A$ pairs. The modified $\mathrm{KdV}\left(\mathrm{mKdV}=\mathrm{m}^{1} \mathrm{KdV}\right), \mathrm{m}^{2} \mathrm{KdV}$ and $\mathrm{m}^{3} \mathrm{KdV}$ equations were thus constructed, the second of which can be reduced to the exponential CalogeroDegasperis (CD) equation by an exponential point change of variables and the third contains the elliptic CD equation. The only price we have to pay for this simplicity is a rapidly growing amount of calculation.

Despite its apparent simplicity, the method of dressing chains is an extremely powerful method, as can be illustrated in following example (see details in [2-4]): as noted in [2] and [3], the $\mathrm{m}^{\mathrm{n}} \mathrm{KdV}$ equations $(\mathrm{n}=0, \ldots, 3)$, together with the Krichever-Novikov equation, exhaust all the integrable equations of the form $u_{t}+u_{x x x}+f\left(u_{x x}, u_{x}, u\right)=0$. In [4] we generalized the dressing-chain method to considerably $(1+2)$-dimensional nonlinear equations: the Kadomtsev-Petviashvili (KP) and the Boiti-Leon-Pempinelli (BLP) equations. A new result, which is characteristic precisely of multidimensional equations, is the discovery of two types of dressing chains for the KP equation (we call these the conjugate chains). The new chain can be constructed with the help of binary DT. 
The aim of this work is the generalization of the dressing chains method for the nonlinear Schrödinger (NLS) and Davey-Stewartson (DS) equations. The plan of this paper is as follows. In Sections 2 and 3 we review the dresing chains method for the higher and lower KdV equations and for the higher KP equations. The dressing chains for the NLS and for the DS equations is discussed in Sections 4, 5. In Sec. 6 we develop a technique for constructing of exact localized solution of DS equations with a reduction constraint imposed and give some of these solutions. We conclude with a discussion on possible role of discrete symmetry's dressing chains in the theory of integrable PDE.

And last but not least. The dressing chains method allow us to construct new integrable equations and, in the other hand, to find a links between known ones. For example, the sine-Gordon, $\mathrm{KdV}$, and $\mathrm{CD}$ equations can be constructed starting out from the KdV equation via dressing chains. Maybe it is only one integrable equation really?

\section{$2 \quad 2 \mathrm{D}-\mathrm{P}_{4}$ equation}

Let us begin our discussion of the Borisov-Zykov method by analysing the simplest of all possible integrable systems, the KdV equation. The starting point is the $L A$ pair for the KdV equation

$$
\psi_{x x}=(u-\lambda) \psi, \quad \psi_{t}=2(u+2 \lambda) \psi_{x}-u_{x} \psi,
$$

where the KdV equation

$$
u_{t}-6 u u_{x}+u_{x x x}=0 .
$$

Setting $\tau=\phi_{x} / \phi$, where $\phi$ is a partial solution of (2) with the $\lambda=\mu$ we can write the $L A$ pair as

$$
\tau_{x}=-\tau^{2}+u-\mu, \quad \tau_{t}=\left[2(u+2 \mu) \tau-u_{x}\right]_{x} .
$$

Exepting $u$ from (4) we get

$$
\tau_{t}=6 \tau^{2} \tau_{x}-\tau_{x x x}+6 \mu \tau_{x} .
$$

If $\mu=0$ then we have the well-known mKdV equation. To construct $L A$ pair for the (4) we use the DT,

$$
u_{1}=u-2 \tau_{x}, \quad \psi_{1}=\psi_{x}-\tau \psi .
$$

Setting $\sigma=\psi_{1, x} / \psi_{1}$, we get the x-chain

$$
(\sigma+\tau)_{x}=-\sigma^{2}+\tau^{2}-\lambda+\mu .
$$

and t-chain,

$$
(\sigma+\tau)_{t}=\left[2\left(-\tau_{x}+\tau^{2}+\mu+2 \lambda\right) \sigma-2 \tau \tau_{x}+6 \mu \tau+2 \tau^{3}\right]_{x} .
$$

At last, after introduction of the auxiliary function (denoted by $\Psi$ ),

$$
\sigma+\tau=\Psi
$$

we obtain the $L A$ pair for the (4)

$$
\Psi_{x}=-\Psi^{2}+2 \tau \Psi-\lambda+\mu, \quad \Psi_{t}=2\left[\left(\tau^{2}-\tau_{x}+\mu+2 \lambda\right) \Psi+2(\mu-\lambda) \tau\right]_{x} .
$$


Starting out from the (6) we can find the $\mathrm{m}^{2} \mathrm{KdV}$ (which be reduced to the exponential CD equation by an exponential point change of variables) with it's $L A$ pair. Continuining this procedure, we obtain the elliptic CD equation $\left(\mathrm{m}^{3} \mathrm{KdV}\right)$ and it is the end of this way. The $\mathrm{m}^{3} \mathrm{KdV}$ equation is the last equation which can be obtained by this method.

At first, we may wonder why the limitation appears by the $\mathrm{n}=3$. However, we can write the $L A$ pairs for the $\mathrm{m}^{\mathrm{n}} \mathrm{KdV}$ with $n<4$ as two Riccati equations. It is incorrectly for the $\mathrm{m}^{4} \mathrm{KdV}[2]$. This equation, therefore, is different from the ones found earlier and cannot be used by the old scheme. In the other hand, as we have mentioned in Introduction, the $\mathrm{m}^{\mathrm{n}} \mathrm{KdV}$ equations $(\mathrm{n}=0, \ldots, 3)$, together with the Krichever-Novikov equation, exhaust all the integrable equations of the form $u_{t}+u_{x x x}+f\left(u_{x x}, u_{x}, u\right)=0$. So this limitation is something behind that. We can understand the triviality of this limitation with the help of the Painleve property. Let consider the $\mathrm{m}^{1} \mathrm{KdV}$ equation (4). The function $\tau$ is the solution of both this equation and two Riccati equations (3) (inserting $\tau_{x}$ from the first equation (3) in the second one we obtain the second Riccati equation), so it is obvious that the function $\tau$ has the Painleve property and that it is incorrectly for the solutions of the $\mathrm{m}^{3} \mathrm{KdV}$ with $n>3$.

In [5] the continuation of the $\mathrm{KdV}$ equation hierarchy in direction corresponding to the negative power of the spectral parameter is constructed by the following way: let the L-operator has the form (1),

$$
L=-\partial_{x}^{2}+u(x, t)
$$

and the nonlinear equations are

$$
L_{t}=\left[L, A_{N}\right]
$$

The operators $A_{N}$ have the form

$$
A_{N}=\sum_{m=-1}^{N} K_{m}(L)^{m}
$$

where $N=-1,-2, \ldots$ and $K_{m}$ are some operators.

The first lower $\mathrm{KdV}(\mathrm{N}=-1)$ equation has the form (see [5]),

$$
\mathrm{KdV}_{-1}(\sigma) \equiv\left(\sigma_{x}^{2}+\sigma_{x x}\right)_{t}-\left(e^{2 \sigma}\right)_{x}=0,
$$

where $\sigma$ is connected with $u$ by the following way

$$
u=-\sigma_{x x}-\sigma_{x}^{2} .
$$

Let $\sigma=i q / 2$, where $q=q(x, t)$. It easy to see that

$$
\mathrm{KdV}_{-1}(\sigma)=\frac{1}{2}\left(i \partial_{x}-q_{x}\right)\left(q_{x t}-2 \sin q\right)=0,
$$

so we have the Miura transformation between the $\mathrm{KdV}_{-1}$ equation and the sine-Gordon equation.

LA-pair for the (7) has the form,

$$
\psi_{x x}=\left(\frac{i q_{x x}}{2}-\frac{q_{x}^{2}}{4}+\lambda^{2}\right) \psi, \quad \psi_{t}=\frac{1}{2 \lambda^{2}} e^{i q}\left(\psi_{x}-\frac{i q_{x}}{2} \psi\right),
$$

Starting out from the (8) we can find the LA-pair for the sine-Gordone equation. To do this we introduce new function $\tilde{\psi}$

$$
\tilde{\psi}=\frac{1}{\lambda}\left(\psi_{x}-\frac{i}{2} q_{x} \psi\right) .
$$


therefore

$$
\tilde{\psi}_{x}=\lambda \psi-\frac{i}{2} q_{x} \tilde{\psi}, \quad \psi_{t}=\frac{1}{2 \lambda} e^{i q} \tilde{\psi}, \quad \tilde{\psi}_{t}=-\frac{1}{2 \lambda}\left(i q_{x t}-e^{i q}\right) \psi
$$

Substituting $2 \sin q$ in place of $q_{x t}$ (in the eq. for the $\tilde{\psi}_{t}$ ) we can see that $(9),(10)$ are the well known LA-pair for the sine-Gordon equation.

Thus, we can use the method of dressing chains to both higher and lower KdV equations. The sine-Gordon, $\mathrm{KdV}$, and $\mathrm{CD}$ equations can be constructed starting out from the $\mathrm{KdV}$ equation via dressing chains of discrete symmetries. There are simply different representations of the same equation. From this this standpoint, the difference between sine-Gorgon, $K d V, m K d V$ and $C D$ equations is similar to the difference between the Maxwell equations in different gauges!

Surprisingly, much of the analysis of the simple system carries over directly to the multidimensional case. The KP equations are given by

$$
u_{t}+6 u u_{x}+u_{x x x}=-3 \alpha^{2} v_{y}, \quad v_{x}=u_{y},
$$

where $u=u(x, y, t), v=v(x, y, t), \alpha^{2}= \pm 1$. The $L A$ pair for the KP equations is:

$$
\alpha \psi_{y}+\psi_{x x}+u \psi=0, \quad \psi_{t}+4 \psi_{x x x}+6 u \psi_{x}+3\left(u_{x}-\alpha v\right) \psi=0 .
$$

Setting $f \equiv \log \phi, \tau \equiv f_{x}$, where $\phi$ is a partial solution of (11), we can write $L A$ pair (11) as

$$
\begin{aligned}
& \alpha \tau_{y}+\left(\tau_{x}+\tau^{2}+u\right)_{x}=0 \\
& \tau_{t}+\left(4 \tau_{x x}+12 \tau \tau_{x}+4 \tau^{3}+6 u \tau+3 u_{x}-3 \alpha v\right)_{x}=0 .
\end{aligned}
$$

It follows from the first equation in (12) that

$$
u=-\tau_{x}-\tau^{2}-\alpha F, \quad F_{x}=\tau_{y} .
$$

Substituting (12) into the second equation in (13) and making some simple transformations, we obtain the well-known mKP equation [6]

$$
\tau_{t}-6 \tau^{2} \tau_{x}+\tau_{x x x}=3 \alpha\left(2 \tau_{x} F-\alpha F_{y}\right), \quad F_{x}-\tau_{y}=0 .
$$

We now construct dressing chains of discrete symmetries. The KP equation admits the Darboux transformation [1]

$$
\psi_{1}=\psi-\tau \psi, \quad u_{1}=u+2 \tau_{x}, \quad v_{1}=v+2 \tau_{y} .
$$

Setting $s \equiv \log \psi_{1}, \sigma=s_{x}$, we see that $\sigma$ satisfies the system of equations obtained from (12) by replacing $u \rightarrow u_{1}$ and $v \rightarrow v_{1}$. Comparing these equations with (12) and eliminating the potentials $u$ and $v$, we obtain

$$
\begin{aligned}
& \alpha(s-f)_{y}+(s+f)_{x x}+s_{x}^{2}-f_{x}^{2}=0, \\
& (s-f)_{t}+\left[2(2 s+f)_{x x}+6 s_{x}^{2}-3 f_{x}^{2}\right]_{x}+4 s_{x}^{3}+6\left(f_{x x}-f_{x}^{2}-\alpha f_{y}\right) s_{x}- \\
& 6 \alpha\left(f_{x y}-f_{x} f_{y}\right)+2 f_{x}^{3}=0 .
\end{aligned}
$$


The first equation in (16) determines the $y$ - chain and the second determines the $t$-chain, denoted by $C_{y}^{(+)}$and $C_{t}^{(+)}$respectively. Later (in Sec. 3) we will generalize the BorisovZykov approach for the case of the KP equation, and we will find that the results of Borisov-Zykov carry over with only one important changes.

As we seen, the Painleve property is connected with the dressing chains. The aproach of using dressing chain (5) to construct the $P_{4}$ equation was proposed in [7]. It is interesting to apply this method to the chain (16).

To do this it is helpful to incert the periodic condition. Well known that the periodic condion for the (5) led to the $P_{4}$ equations. Let consider the dressing chain $C_{y}^{(+)}(16)$. Setting $f=f_{n}, s=f_{n+1}$ we use the condition of periodic

$$
f_{n+N}=f_{n}+c(y) \text {. }
$$

It easy to see that

$$
u_{n+N}=u_{n}+\alpha c^{\prime},
$$

so the condition (17) get us some generalization of the harmonic oscillator. We choose $N=3$ and $c=-2 y / \alpha$. Introducing new fields $g_{n}, n=1,2,3$

$$
f_{1}=\frac{1}{2}\left(g_{1}-g_{2}+g_{3}+c\right), \quad f_{2}=\frac{1}{2}\left(g_{1}+g_{2}-g_{3}-c\right), \quad f_{3}=\frac{1}{2}\left(-g_{1}+g_{2}+g_{3}+c\right),
$$

we can now rewrite the equations for the $f_{n}$ by the following way

$$
\begin{gathered}
\alpha\left(g_{2 y}-g_{3 y}-c_{y}\right)+\left(g_{2 x}-g_{3 x}\right) g_{1_{x}}+g_{1_{x x}}=0 \\
\alpha\left(-g_{1_{y}}+g_{3 y}+c_{y}\right)+\left(g_{3 x}-g_{1_{x}}\right) g_{2 x}+g_{2 x x}=0 \\
\alpha\left(g_{1_{y}}-g_{2 y}-c_{y}\right)+\left(g_{1_{x}}-g_{2 x}\right) g_{3 x}+g_{3 x x}=0 .
\end{gathered}
$$

Excluding $g_{3}$, and using the compatibility condition $D_{y} g_{2, x x}=D_{x}^{2} g_{2, y}$ we get the nonlinear equation which can be written via some transformation as

$$
\begin{aligned}
& z_{x x}=\frac{1}{2} \frac{z_{x}^{2}}{z}+\frac{3}{2} z^{3}+4 x z^{2}+2\left(x^{2}-2\right) z+\frac{\beta}{z}+ \\
& +\frac{3 \alpha^{2} q^{2}}{2 z}-3 \alpha q z+\frac{\alpha}{z} D_{x}^{-1} D_{y}\left(z^{3}+2 x z^{2}-3 \alpha q z\right), \quad z_{y}=q_{x} .
\end{aligned}
$$

where $z=g_{1 x}$ and $q=g_{1 y}$. The one-dimensional limit (where $D_{y}=q=0$ ) of Eq. (18) is the $P_{4}$ equation, so the (18) is the two-dimensional generalization of the $P_{4}$ equation and it should be called the $2 \mathrm{D}-\mathrm{P}_{4}$ equations.

The price we pay for the $\mathrm{D}=2$ is that locality in $x$ is lost. That is in the usual run of things for the multidimensional integrable systems.

\section{Conjugate chains for the KP equations}

The correspondence between the KdV dressing chains formalism that we develop in the previous section and the KP dressing chains formalism is quite remarkable. We find that almost the entire $\mathrm{KdV}$ formalism can be imported into the KP chains. 
Chains (16) involve two $L A$ pairs for Eqs. (14), which arise after introducting the auxiliary function (denoted by $\Psi$ ) according to one of two rules,

$$
\Psi=s-f, \quad \Psi=s+f .
$$

Using the first rule, we obtain

$$
\begin{aligned}
& \alpha \Psi_{y}+(2 f+\Psi)_{x x}+2 f_{x} \Psi_{x}+\Psi_{x}^{2}=0, \\
& \Psi_{t}+2\left(2 \Psi_{x x}+6 f_{x} \Psi_{x}+3 \Psi_{x}^{2}+3 \theta\right)_{x}+6 \theta \Psi_{x}+4(3 f+\Psi)_{x} \Psi_{x}^{2}=0, \\
& \theta \equiv f_{x x}+f_{x}^{2}-\alpha f_{y}
\end{aligned}
$$

from (16). The compatibility condition for these equations is

$$
\left(f_{t}-2 f_{x}^{3}+f_{x x x}\right)_{x}=3 \alpha\left(2 f_{x x} f_{y}-\alpha f_{y y}\right)
$$

which is merely another form of the mKP equation (for $\tau=f_{x}$ and $F=f_{y}$ ).

This process can be repeated. In particular, the system (20) contains a new nonlinear equation obtained by eliminating the potential $f$. For this purpose, we linearize the first equation in (20) by the substituting

$$
f=-\frac{1}{2}(\Psi+\alpha \xi)
$$

where $\xi=\xi(x, y, t)$. Inserting (22) in the second equation in $(20)$, differentiating it with respect to $x$, and introducing $S \equiv \Psi_{x}$, we obtain the system

$$
\begin{aligned}
& S_{t}+S_{x x x}-\frac{3}{2} S^{2} S_{x}=-3 \alpha^{2}\left[\left(\frac{1}{2} \xi_{x}^{2}+\xi_{y}\right) S+\left(\frac{1}{2} \xi_{x}^{2}+\xi_{y}\right)_{x}\right]_{x}, \\
& S_{y}=\left(\xi_{x x}+\xi_{x} S\right)_{x},
\end{aligned}
$$

Although Eqs. (23) look like a two-dimensional generalization of the mKdV equation, the one-dimensional limit (where $\partial_{y}=0$ ) of $(20)$ is

$$
g_{t}+\left(g_{x x}-\frac{\alpha^{2}}{2} g^{3}-\frac{3}{2} \frac{g_{x}^{2}}{g}\right)_{x}=0,
$$

(where $g(x, t) \equiv \xi_{x}=\exp (-\Psi)$ ) rather than the mKdV equation. After an exponential point change of variable, this equation reduced to the exponential CD equation. Therefore, Eq. (23) should be called the two-dimensional CD equation rather than the two-dimensional $\mathrm{mKdV}$ equation.

In [2], another representation for $\Psi$ was used in the derivation of the CD equation, namely, the representation determined by the second formula in (19). We have used this representation in [4].

In addition to the usual DT, the KP equations admit the so-called binary Darboux transformations [1], which, as we now show, allow us to construct new KP dressing chains.

It is obvious that the KP equation admits the $L A$ pair

$$
-\alpha \chi_{y}+\chi_{x x}+u \chi=0, \quad \chi_{t}+4 \chi_{x x x}+6 u \chi_{x}+3\left(u_{x}+\alpha v\right) \chi=0
$$


which can be obtained from (11) by simple replacing $\alpha \rightarrow-\alpha$. Let

$$
\begin{aligned}
& d Q(\chi, \psi)=\chi \psi d x+\frac{1}{\alpha}\left(\chi_{x} \psi-\chi \psi_{x}\right) d y+4\left(\psi_{x} \chi_{x}-\chi_{x x} \psi-\chi \psi_{x x}-\frac{3}{2} u \chi \psi\right) d t \\
& Q(\chi, \psi) \equiv \int d Q(\chi, \psi) .
\end{aligned}
$$

It is easy to see that the one-form $d Q(\chi, \psi)$ is closed if $\psi$ and $\chi$ are solutions of Eqs. (11) and (24). Pair (24) also admits the Darboux transformation

$$
\chi_{-1}=\chi-\rho \chi, \quad u_{-1}=u+2 \rho_{x}, \quad v_{-1}=v+2 \rho_{y},
$$

where $\rho=(\log \tilde{\chi})_{x}$, and $\tilde{\chi}$ is a partial solution of (24). Applying DT (15), we have

$$
\chi_{1}=\frac{A+B Q(\chi, \psi)}{\psi}
$$

where $A$ and $B$ are arbitrary constants. Now using (25), we obtain

$$
u_{1,-1}=u+2[\log (A+B Q(\chi, \psi))]_{x x}, \quad v_{1,-1}=v+2[\log (A+B Q(\chi, \psi))]_{x y} .
$$

We set $\sigma \equiv \chi_{1, x} / \chi_{1}$. This function satisfies the equation obtained from (12) by replacing $u \rightarrow u_{1}, v \rightarrow v_{1}$ and $\alpha \rightarrow-\alpha$. In other words, this yield new integrable $y$ - and $t$-chains $\left(C_{y}^{(-)}\right.$and $\left.C_{t}^{(-)}\right)$, which can be called conjugate to the $C^{(+)}$chains discussed above:

$$
\begin{aligned}
& -\alpha(s+f)_{y}+(s+f)_{x x}+s_{x}^{2}-f_{x}^{2}=0, \\
& (s+f)_{t}+\left[4(s+f)_{x x}+6 s_{x}^{2}-3 f_{x}^{2}\right]_{x}+4 s_{x}^{3}+6\left(f_{x x}-f_{x}^{2}-\alpha f_{y}\right) s_{x}-6 \alpha f_{x} f_{y}-2 f_{x}^{3}=0,
\end{aligned}
$$

where we still have $\tau=f_{x}$ and $\sigma=s_{x}$. Comparing the expressions for $C^{(-)}$and $C^{(+)}$ from (16), we see that these chains do not reduce to each other, thereby justifying the term "conjugate". This implies that the chains $C^{(-)}$lead to new integrable equations and also to the corresponding $L A$ pairs. To construct these, we should again use two possibilities to define $\Psi$, see Eq. (19). In the present case, it is convenient to choose the second formula. As the result, we obtain yet another $L A$ pair for the mKP equation (21):

$$
\begin{aligned}
& -\alpha \Psi_{y}+\Psi_{x x}-2 f_{x} \Psi_{x}+\Psi_{x}^{2}=0, \\
& \Psi_{t}+2\left(2 \Psi_{x x}-3 f_{x} \Psi_{x}+3 \Psi_{x}^{2}\right)_{x}+6\left(f_{x}^{2}-\alpha f_{y}\right) \Psi_{x}-6 f_{x} \Psi_{x x}+4(\Psi-3 f)_{x} \Psi_{x}^{2}=0 .
\end{aligned}
$$

Linearizing the first equation by the substitution that differs from the (22) only by the sign of the right-hand side and defined $S \equiv \Psi_{x}$, we get

$$
\begin{aligned}
& S_{t}+4 S_{x x x}-\frac{3}{2} S^{2} S_{x}=3 \alpha\left[\xi_{x x} S+2 \xi_{x} S_{x}+\alpha\left(\xi_{y}-\frac{1}{2} \xi_{x}^{2}\right) S\right]_{x}, \\
& \alpha S_{y}=\left(S_{x}-\alpha \xi_{x} S\right)_{x} .
\end{aligned}
$$

Equations (26) are similar to (23) and also reduce to the CD equation in the onedimensional limit (for $\alpha^{2}=1$ ). At the same time, as follows from the method of their construction, Eqs. (26) do not reduce to system (23) via a 1:1 change of dependent variables (by a gauge transformation); therefore, Eqs. (26) can be called conjugate to the Eqs. (23). 


\section{Dressing chains for the NLS equation}

The results in last section indicates that generalizing the dressing-chain method to considerably more complicated NLS and DS equations can be very fruitful. In this section we use the dressing chains of discrete symmetries to proliferate the NLS equation. Recently, Shabat has studied such chains for the Zakharov-Shabat spectral problem in some unusual gauge [8]. We have another aim: to construct higher mNLS equations. We can do it in two gauges, each with its own advantages and disadvantages:

(1) Standart symmetric Zakharov-Shabat gauge. In this gauge we have very clear connections between NLS and $\mathrm{m}^{\mathrm{n}} \mathrm{NLS}$ equations. The disadvantage of this gauge is a very rapidly growing ammount of calculations. For the $n>2$ we obtain very cumbersome equations.

(2) New Shabat gauge [8]. The advantage of this gauge is that equations has more compact form. However, the connection between real NLS and real mNLS equations is not obvious in this gauge. The reconstruction of the $\mathrm{m}^{\mathrm{n}} \mathrm{NLS}$ for a $n>2$ must be checked tediously.

Here, we choose the standard gauge for the spectral problem and therefore the $L A$ pair for the NLS equation is

$$
\Psi_{t}=-2 i \sigma_{3} \Psi \Lambda^{2}+2 i U \Psi \Lambda+V \Psi, \quad \Psi_{x}=-i \sigma_{3} \Psi \Lambda+i U \Psi
$$

where

$$
U=\left(\begin{array}{cc}
0 & u \\
v & 0
\end{array}\right), \quad \sigma_{3}=\left(\begin{array}{cc}
1 & 0 \\
0 & -1
\end{array}\right), \quad V=\sigma_{3}\left(i U^{2}-U_{x}\right),
$$

and $\Lambda$ is arbitrary constant matrix. The compatibility condition get us the NLS equations

$$
i u_{t}+u_{x x}+2 u^{2} v=0, \quad-i v_{t}+v_{x x}+2 v^{2} u=0 .
$$

Let $\Phi$ is a partial solution of $(27)$ with the $\Lambda=\operatorname{diag}((\lambda+\mu) / 2,(\lambda-\mu) / 2), \Psi$ is the same for the $\Lambda_{1}=\operatorname{diag}\left(\left(\lambda_{1}+\mu_{1}\right) / 2,\left(\lambda_{1}-\mu_{1}\right) / 2\right)$ and $\tau \equiv \Phi \Lambda \Phi^{-1}$. This matrix function is the solution of the system

$$
\tau_{x}=i\left[\tau, \sigma_{3}\right] \tau+i[U, \tau], \quad \tau_{t}=2 \tau_{x} \tau+[V, \tau]
$$

The Darboux transformation has the form

$$
\Psi \rightarrow \Psi_{1}=\Psi \Lambda_{1}-\tau \Psi, \quad U \rightarrow U_{1}=U+\left[\tau, \sigma_{3}\right]
$$

There are two discrete symmetries of the Zakharov-Shabat spectral problem: S- and Tsymmetries. The transformation (29) is $S^{2}$ symmetry. We must use squared S-symmetry because an elementary S-symmetries are unsufficient to construct exact solutions of the NLS.

Using (29) we obtain

$$
u \rightarrow u_{1}=u-2 b, \quad v \rightarrow v_{1}=v+2 c,
$$

where

$$
\tau=\left(\begin{array}{ll}
a & b \\
c & d
\end{array}\right)
$$


Substituting (31) into (28) we get

$$
\begin{gathered}
a_{x}=-d_{x}=-i(2 b c-u c+v b), \quad b_{x}=-i(2 b d+(a-d) u), \\
c_{x}=i(2 a c+(a-d) v)
\end{gathered}
$$

and

$$
\begin{gathered}
a_{t}=\left(a^{2}\right)_{x}+2 b_{x} c-b v_{x}-c u_{x}, \quad d_{t}=\left(d^{2}\right)_{x}+2 b c_{x}+b v_{x}+c u_{x} \\
b_{t}=2\left(i b u v+a_{x} b+b_{x} d\right)+(a-d) u_{x}, \quad c_{t}=2\left(-i c u v+a c_{x}+c d_{x}\right)+(a-d) v_{x} .
\end{gathered}
$$

Calculating the determinant and trace of matrix $\tau$ we get

$$
a d-b c=\frac{\lambda^{2}-\mu^{2}}{4}, \quad a+d=\lambda .
$$

Using (34) we get

$$
a=\frac{1}{2}\left(\lambda \pm \sqrt{\mu^{2}-4 b c}\right) .
$$

Eliminating $u$ and $v$ from the last two Eqs. (32) and putting everything together we find mNLS $\left(\mathrm{m}^{1} \mathrm{NLS}\right)$ equation

$$
\begin{gathered}
\left(\mu^{2}-4 b c\right)\left(i b_{t}+b_{x x}-2 b^{2} c\right)+2 \lambda\left(\lambda c+2 i c_{x}\right) b^{2}+2\left(b_{x} c+2 b c_{x}\right) b_{x} \equiv\left(\mu^{2}-4 b c\right) \beta(b, c)=0, \\
\left(\mu^{2}-4 b c\right)\left(-i c_{t}+c_{x x}-2 c^{2} b\right)+2 \lambda\left(\lambda b-2 i b_{x}\right) c^{2}+2\left(b c_{x}+2 b_{x} c\right) c_{x} \equiv\left(\mu^{2}-4 b c\right) \gamma(b, c)=0 .
\end{gathered}
$$

The designeshions $\beta(b, c)$ and $\gamma(b, c)$ will be useful (see (41), (42)).

As we have mentioned above, the NLS equation has a broader set of symmetries. In addition to the Darboux transformations, the discrete symmetries include the Schlesinger transformations or T-symmetries. The T-symmetries produce an explicity invertible Bäcklund auto-transformations for the NLS equation

$$
u \rightarrow u_{1}=u_{x x}+u^{2} v-\frac{u_{x}^{2}}{u}, \quad v \rightarrow v_{1}=\frac{1}{u}
$$

and

$$
u \rightarrow u_{-1}=\frac{1}{v}, \quad v \rightarrow v_{-1}=v_{x x}+v^{2} u-\frac{v_{x}^{2}}{v}
$$

where

$$
\left(u_{1}\right)_{-1}=\left(u_{-1}\right)_{1}=u, \quad\left(v_{1}\right)_{-1}=\left(v_{-1}\right)_{1}=v .
$$

These symmetries are related to the Toda chain and some of its generalizations [9].

The mNLS equation (36) has the similar property. We have very cumbersome Schlesinger transformations for the (36) so we show ones when $\lambda=\mu=0$. In this case, the (36) has an elegant form

$$
\begin{aligned}
& i b_{t}+b_{x x}-2 b^{2} c-\frac{b_{x} c_{x}}{c}-\frac{1}{2} \frac{b_{x}^{2}}{b}=0, \\
& -i c_{t}+c_{x x}-2 b c^{2}-\frac{b_{x} c_{x}}{b}-\frac{1}{2} \frac{c_{x}^{2}}{c}=0 .
\end{aligned}
$$


The explicity invertible Bäcklund auto-transformations for the (37) are

$$
\begin{aligned}
& b \rightarrow b_{1}=\frac{1}{4} \frac{\left(2 c b_{x}^{2}-2 c b b_{x x}+c_{x} b_{x} b+4 c^{2} b^{3}\right)^{2}}{c^{2} b\left(b_{x}^{2}-4 c b^{3}\right)}, \quad c \rightarrow c_{1}=\frac{4 c b^{2}}{b_{x}^{2}-4 c b^{3}}, \\
& b \rightarrow b_{-1}=\frac{4 b c^{2}}{c_{x}^{2}-4 b c^{3}}, \quad c \rightarrow c_{-1}=\frac{1}{4} \frac{\left(2 b c_{x}^{2}-2 b c c_{x x}+b_{x} c_{x} c+4 b^{2} c^{3}\right)^{2}}{b^{2} c\left(c_{x}^{2}-4 b c^{3}\right)} .
\end{aligned}
$$

It is easy to verify that

$$
\left(b_{1}\right)_{-1}=\left(b_{-1}\right)_{1}=b, \quad\left(c_{1}\right)_{-1}=\left(c_{-1}\right)_{1}=c .
$$

To obtain dressing chains of discrete symmetries $S^{2}$ we construct new matrix function $\tau_{1}=\Psi_{1} \Lambda_{1} \Psi_{1}^{-1}$ with $\Psi_{1}$ from the (29). Its elements $a_{1}, b_{1}, c_{1}$ and $d_{1}$ are solutions of (32)-(35) by replacing $\lambda \rightarrow \lambda_{1}, \mu \rightarrow \mu_{1}, u \rightarrow u_{1}$ and $v \rightarrow v_{1}$ (see (30)). Eliminating potentials $u$ and $v$, we obtain our chains

$$
\begin{aligned}
& (\lambda-2 a) b_{1, x}-\left(\lambda_{1}-2 a_{1}\right) b_{x}+2 i\left(\lambda_{1}-a_{1}\right)(\lambda-2 a) b_{1}-2 i\left(\lambda_{1}-2 a_{1}\right) a b=0, \\
& (\lambda-2 a) c_{1, x}-\left(\lambda_{1}-2 a_{1}\right) c_{x}-2 i(\lambda-2 a) a_{1} c_{1}+2 i\left(\lambda_{1}-2 a_{1}\right)(\lambda-a) c=0, \\
& b_{1, t} b-b_{1} b_{t}+2\left[(\lambda-a) b_{1} b_{x}-\left(\lambda_{1}-a_{1}\right) b_{1, x} b\right]+\left(\lambda_{1}-2 a_{1}\right)\left(b^{2}\right)_{x}+K_{1} b_{1}+M_{1} b=0, \\
& c_{1, t} c-c_{1} c_{t}+2\left(a c_{1} c_{x}-a_{1} c_{1, x} c\right)+\left(\lambda_{1}-2 a_{1}\right)\left(c^{2}\right)_{x}+K_{2} c_{1}+M_{2} c=0,
\end{aligned}
$$

where

$$
\begin{aligned}
& K_{1}=4 i C(2,2) b^{2}+2\left[\left(a-a_{1}\right)_{x}+2 i B(-2,-2) c\right] b-(\lambda-2 a) B_{x}(-2,-2), \\
& K_{2}=-4 i B(0,2) c^{2}-2\left[\left(a-a_{1}\right)_{x}+2 i C(0,-2) b\right] c-(\lambda-2 a) C_{x}(0,-2), \\
& M_{1}=\left(\lambda_{1}-2 a_{1}\right) B_{x}(-2,-2), \quad M_{2}=\left(\lambda_{1}-2 a_{1}\right) C_{x}(0,-2), \\
& B(n, k)=\frac{i b_{x}+(n \lambda-k a) b}{\lambda-2 a}, \quad C(n, k)=\frac{i c_{x}+(n \lambda-k a) c}{\lambda-2 a},
\end{aligned}
$$

and fields $a, d\left(a_{1}, d_{1}\right)$ are expressed in term of $b, c\left(b_{1}, c_{1}\right)$ via (34)-(35).

Chains (38) involve $L A$ pair for the mNLS (36), which arise after introducing the auxiliary fields

$$
\psi \equiv \frac{b_{1}}{b}, \quad \phi \equiv \frac{c_{1}}{c}, \quad A \equiv a_{1} .
$$

After some calculations we obtain

$$
\begin{aligned}
& \psi_{x}=\left(2 i\left(A-\lambda_{1}\right)-\frac{b_{x}}{b}\right) \psi+\frac{i\left(2 A-\lambda_{1}\right)}{b} B(0,2), \\
& \phi_{x}=\left(2 i A-\frac{c_{x}}{c}\right) \phi+\frac{i\left(2 A-\lambda_{1}\right)}{c} C(2,2) \\
& \psi_{t}=2\left(\lambda_{1}-A\right) \psi_{x}-4 i b c \phi \psi^{2}-2 i(b C(2,2) \psi+c B(0,2) \phi) \psi+P \psi+\frac{\lambda_{1}-2 A}{b} B_{x}(0,2), \\
& \phi_{t}=2 A \phi_{x}+4 i b c \psi \phi^{2}+2 i(b C(2,2) \psi+c B(0,2) \phi) \phi+Q \phi-\frac{\lambda_{1}-2 A}{c} C_{x}(2,2), \\
& A_{x}=-i[2 b c \phi \psi+b C(2,2) \psi+c B(0,2) \phi] \\
& A_{t}=-4 i \lambda_{1} b c \phi \psi-b\left[2 i A C(2,2)+C_{x}(2,2)\right] \psi+c\left[2 i\left(A-\lambda_{1}\right) B(0,2)+B_{x}(0,2)\right] \phi,
\end{aligned}
$$


where

$$
\begin{aligned}
& P=\frac{\left[2 b_{x}\left(\lambda_{1}-A\right)-i b_{x x}\right]\left(\mu^{2}-4 b c\right)+2(b c)_{x}\left[2 b(\lambda-a)-i b_{x}\right]}{b\left(\mu^{2}-4 b c\right)}, \\
& Q=\frac{\left(2 c_{x} A+i c_{x x}\right)\left(\mu^{2}-4 b c\right)+2(b c)_{x}\left(2 c a+i c_{x}\right)}{c\left(\mu^{2}-4 b c\right)} .
\end{aligned}
$$

The mNLS equation (36) arise from the compatibility condition of (39):

$$
\left(\psi_{x}\right)_{t}=\left(\psi_{t}\right)_{x}, \quad\left(\phi_{x}\right)_{t}=\left(\phi_{t}\right)_{x}, \quad\left(A_{x}\right)_{t}=\left(A_{t}\right)_{x}
$$

From the first two equations (40) we get two nonlinear equations

$$
\beta_{1}(b, c)=0, \quad \gamma_{1}(b, c)=0 .
$$

The connection between these equations and the mNLS (36) is given by

$$
\beta_{1}(b, c)=b\left(\frac{\beta(b, c)}{b}\right)_{x}, \quad \gamma_{1}(b, c)=c\left(\frac{\gamma(b, c)}{c}\right)_{x},
$$

so we have not (36) but

$$
\beta(b, c)=m b, \quad \gamma(b, c)=m^{\prime} c,
$$

where $m m^{\prime}$ are arbitrary constants. Substituting $b_{t}$ and $c_{t}$ (which are expressed from (42)) into the third equation of the system (40) we obtain $m^{\prime}=m$. Thus, using the gauge transformation

$$
b \rightarrow e^{-i m t} b, \quad c \rightarrow e^{i m t} c,
$$

we can reduce the (42) to mNLS (36)

$$
\beta(b, c)-m b \rightarrow e^{-i m t} \beta(b, c)=0, \quad \gamma(b, c)-m c \rightarrow e^{-i m t} \gamma(b, c)=0 .
$$

This completes the proof that the system (39) is the $L A$ pair of mNLS. We are giving two formulas (for convenience of reader) which are useful to check of this $L A$ pair:

$$
a_{x}=\frac{(b c)_{x}}{\lambda-2 a}, \quad a_{t}=\lambda a_{x}+b_{x} c-b c_{x}-b C_{x}(0,-2)+c B_{x}(-2,-2),
$$

We will shortly see the advantage of carefully working out the details of the NLS dressing chains. We will find that almost all of this formalism carried over directly into the more complicated DS dressing chains. Formulaes for the DS dressing chains are very bulki but ones has the same structure.

\section{Conjugate chains of the DS equations}

The DS equations has the form

$$
\begin{aligned}
& i u_{t}+u_{x x}+\frac{1}{\alpha^{2}} u_{y y}-\frac{2}{\alpha^{2}} u^{2} v+q u=0, \quad-i v_{t}+v_{x x}+\frac{1}{\alpha^{2}} v_{y y}-\frac{2}{\alpha^{2}} v^{2} u+q v=0, \\
& q_{y y}-\alpha^{2} q_{x x}=-4(u v)_{x x}
\end{aligned}
$$

where $u=u(x, y, t), v=v(x, y, t), q=q(x, y, t)$. We have the DS-I system if $\alpha=1$ and DS-II, if $\alpha=i$. Under the reduction $v= \pm \bar{u}$, one obtains a known model that describes 
the propogation of a small amplitude wave packet that is quasi-one-dimensional and quasimonochromatic over the surface of a nonviscous curl-free liquid [10]. The quantity $u$ is the envelope of the velocity potential, while $q$ describes the nonlocal flow generated by the wave packet. A different application of (43) is related to the dynamics of plasma waves [11]. We woun't use this reduction restriction in this section.

$L A$ "pair" for the (43) has the form

$$
\begin{aligned}
& \psi_{y}=\alpha \psi_{x}+u \phi, \quad \phi_{y}=-\alpha \phi_{x}+v \psi \\
& \psi_{t}=2 i \psi_{x x}+\frac{2 i}{\alpha} u \phi_{x}+\left(\frac{1}{2}\left[\frac{1}{\alpha} F_{y}+F_{x}\right]-\frac{i}{\alpha^{2}} u v\right) \psi+\frac{i}{\alpha^{2}}\left(\alpha u_{x}+u_{y}\right) \phi \\
& \phi_{t}=-2 i \phi_{x x}+\frac{2 i}{\alpha} v \psi_{x}+\left(\frac{1}{2}\left[\frac{1}{\alpha} F_{y}-F_{x}\right]+\frac{i}{\alpha^{2}} u v\right) \phi+\frac{i}{\alpha^{2}}\left(\alpha v_{x}-v_{y}\right) \psi,
\end{aligned}
$$

where $q=-i F_{x}$. by

Let $\left\{\psi_{1}, \phi_{1} ; \psi_{2}, \phi_{2} ; \psi, \phi\right\}$ are solutions of (44) for the same $u, v$ and $F$. DT is given

$$
\begin{aligned}
& \psi \rightarrow \psi_{1}=\psi_{x}-a \psi-b \phi, \quad \phi \rightarrow \phi_{1}=\phi_{x}-c \psi-d \phi, \\
& u \rightarrow u_{1}=u+2 \alpha b, \quad v \rightarrow v_{1}=v-2 \alpha c, \quad F \rightarrow F_{1}=F+4 i(a+d),
\end{aligned}
$$

where

$$
\begin{array}{ll}
a=\frac{\psi_{1, x} \phi_{2}-\psi_{2, x} \phi_{1}}{\Delta}, & b=\frac{\psi_{2, x} \psi_{1}-\psi_{1, x} \psi_{2}}{\Delta}, \quad c=\frac{\phi_{1, x} \phi_{2}-\phi_{2, x} \phi_{1}}{\Delta}, \\
d=\frac{\phi_{2, x} \psi_{1}-\phi_{1, x} \psi_{2}}{\Delta}, & \Delta=\psi_{1} \phi_{2}-\psi_{2} \phi_{1} .
\end{array}
$$

The quantities $a, b, c d$ are solutions of the system

$$
\begin{aligned}
& a_{y}=\alpha\left(a_{x}+2 b c\right)+u c-v b, \quad b_{y}=\alpha\left(b_{x}+2 b d\right)+(d-a) u+u_{x}, \\
& c_{y}=-\alpha\left(c_{x}+2 a c\right)+(a-d) v+v_{x}, \quad d_{y}=-\alpha\left(d_{x}+2 b c\right)-u c+v b, \\
& a_{t}=2 i\left[\left(a_{x}+2 b c+a^{2}+\frac{q}{4}\right)_{x}+2(a+d) b c\right]+\frac{i}{\alpha^{2}}\left[b v_{y}+c u_{y}-(u v)_{x}\right]+ \\
& +\frac{i}{\alpha}\left[c u_{x}-b v_{x}+2\left(c d u-a b v+(u c)_{x}\right)+\frac{q y}{2}\right], \\
& b_{t}=2 i\left[\left(b_{x}+2 b d\right)_{x}+2\left(a_{x}+b c+d^{2}+\frac{q}{4}\right) b\right]+\frac{i}{\alpha^{2}}\left[u_{x y}+(d-a) u_{y}-2 b u v\right]+ \\
& +\frac{i}{\alpha}\left[u_{x x}+(3 d-a) u_{x}+2\left(d_{x}+d^{2}-a d+b c\right) u-2 b^{2} v\right], \\
& c_{t}=-2 i\left[\left(c_{x}+2 a c\right)_{x}+2\left(d_{x}+b c+a^{2}+\frac{q}{4}\right) c\right]-\frac{i}{\alpha^{2}}\left[v_{x y}+(a-d) v_{y}-2 c u v\right]+ \\
& +\frac{i}{\alpha}\left[v_{x x}+(3 a-d) v_{x}+2\left(a_{x}+a^{2}-a d+b c\right) v-2 c^{2} u\right], \\
& d_{t}=-2 i\left[\left(d_{x}+2 b c+d^{2}+\frac{q}{4}\right)_{x}+2(a+d) b c\right]-\frac{i}{\alpha^{2}}\left[b v_{y}+c u_{y}-(u v)_{x}\right]- \\
& -\frac{i}{\alpha}\left[c u_{x}-b v_{x}+2\left(c d u-a b v-(b v)_{x}\right)-\frac{q y}{2}\right] .
\end{aligned}
$$


These equations can be obtained from the (44). Eqs. (47) are similar to the (13)-(14). The $L A$ pair (44) and Eqs. (47) are simply two different reprersentations of the same equations. To construct mDS equations we must, first, express fields $a$ and $d$ via one function $\theta=\theta(x, y, t)$ defined by the relations

$$
a=\frac{\alpha \theta_{x}+\theta_{y}}{2 \alpha}, \quad d=\frac{\alpha \theta_{x}-\theta_{y}}{2 \alpha} .
$$

It is easy to check the truth of this representation by the substitution (48) into the first and fourth equations (47). Second, we must exclude potentials $u$ and $v$ from the first four equations (47). Introdusing new fields $\xi=\xi(x, y, t), X=\theta_{x}, Y=\theta_{y}$

$$
q=-2 X_{x}-X^{2}-4 b c+\frac{1}{\alpha^{2}}\left(\xi-Y^{2}\right),
$$

and putting everything together we find mDS (mDS-I if $\alpha=1$ and mDS-II if $\alpha=i$ ) equations as the system of five equations for the five functions $b, c, \xi, X$ and $Y$ :

$$
\begin{aligned}
& i b_{t}+2\left(b X+b_{x}\right)_{x}+\frac{1}{\alpha}\left[\left(U X+U_{x}\right)_{x}+2 b(U c-V b)-2 Y\left(b X+b_{x}\right)\right]+ \\
& +\frac{1}{\alpha^{2}}\left[U_{x y}-U\left(X_{y}+X Y\right)-2\left(U_{x} Y+b U V\right)+\xi b\right]+\frac{1}{\alpha^{3}} Y\left(U Y-U_{y}\right)=0, \\
& -i c_{t}+2\left(c X+c_{x}\right)_{x}-\frac{1}{\alpha}\left[\left(V X+V_{x}\right)_{x}-2 c(U c-V b)-2 Y\left(c X+c_{x}\right)\right]+ \\
& +\frac{1}{\alpha^{2}}\left[V_{x y}-V\left(X_{y}+X Y\right)-2\left(V_{x} Y+c U V\right)+\xi c\right]-\frac{1}{\alpha^{3}} Y\left(V Y-V_{y}\right)=0, \\
& i X_{t}+4\left[(b c)_{x}+2 X b c\right]-\frac{2}{\alpha}\left[X(V b-U c)+4 c b_{y}-2(c U)_{x}+b V_{x}-c U_{x}-Y X_{x}\right]- \\
& -\frac{2}{\alpha^{2}}\left[(U V)_{x}-V b_{y}-U c_{y}+Y(b V+c U)\right]-\frac{1}{\alpha^{3}}\left(Y^{2}-\xi\right)_{y}=0, \\
& i Y_{t}+4\left(b_{y} c-b c_{y}\right)+\frac{1}{\alpha}\left[2\left(U_{y} c-U c_{y}+V_{y} b-V b_{y}\right)+\xi_{x}\right]=0, \\
& X_{y}-Y_{x}=0,
\end{aligned}
$$

where

$$
\begin{aligned}
& U=\frac{G_{1}}{2 \alpha\left(2 b c Y+\alpha\left(b c_{x}-b_{x} c\right)\right)}, \quad V=\frac{G_{2}}{2 \alpha\left(2 b c Y+\alpha\left(b c_{x}-b_{x} c\right)\right)}, \\
& G_{1}=\alpha^{3}\left[b_{x} X_{x}-b X_{x x}+2 b\left(2 b c X+b_{x} c-b c_{x}\right)\right]+\alpha^{2}\left[2\left(b c_{y}-b_{y} c\right)-\left(X_{x}+4 b c\right) Y\right] b+ \\
& +\alpha\left(b X_{y y}-b_{x} Y_{y}\right)+b Y Y_{y}, \\
& G_{2}=\alpha^{3}\left[c_{x} X_{x}-c X_{x x}+2 c\left(2 b c X-b_{x} c+b c_{x}\right)\right]+\alpha^{2}\left[2\left(b c_{y}-b_{y} c\right)+\left(X_{x}+4 b c\right) Y\right] c+ \\
& +\alpha\left(c X_{y y}-c_{x} Y_{y}\right)-c Y Y_{y} .
\end{aligned}
$$

As we have mentioned above, the NLS equation has two types of discrete symmetries: Darboux and Schlesinger transformations. In just the same way, the discrete symmetries of the DS equations include T-symmetry. This transformations are given by $([12],[13])$

$$
\begin{aligned}
& u \rightarrow u_{1}=u\left(u v+\alpha^{2}(\log u)_{x x}-(\log u)_{y y}\right), \quad v \rightarrow v_{1}=\frac{1}{u}, \quad q \rightarrow q_{1}=q+4(\log u)_{x x} \\
& u \rightarrow u_{-1}=\frac{1}{v}, \quad v \rightarrow v_{-1}=v\left(u v+\alpha^{2}(\log v)_{x x}-(\log v)_{y y}\right), \quad q \rightarrow q_{-1}=q+4(\log v)_{x x} .
\end{aligned}
$$


The similar transformations for the (49) are given by

$$
\begin{aligned}
& b \rightarrow b_{1}=\frac{M_{1}}{\alpha U(U+2 \alpha b)}, \quad c \rightarrow c_{1}=\frac{b}{U(U+2 \alpha b)}, \\
& X \rightarrow X_{1}=X+2 \alpha \frac{U b_{x}-U_{x} b}{U(U+2 \alpha b)}, \quad Y \rightarrow Y_{1}=\frac{-U^{2} Y+2 \alpha\left[U U_{x}-b U_{y}+\alpha U\left(b_{x}+b X\right)\right]}{U(U+2 \alpha b)}, \\
& \xi \rightarrow \xi_{1}=\xi+4 \alpha \frac{\alpha^{2} U\left(b_{x x}+(b X)_{x}\right)+\alpha\left[b\left(U Y_{x}-U_{x y}\right)+U\left(U_{x x}-b_{x} Y\right)\right]-U U_{x} Y}{U(U+2 \alpha b)},
\end{aligned}
$$

and

$$
\begin{aligned}
& c \rightarrow c_{-1}=\frac{M_{-1}}{\alpha V(V-2 \alpha c)}, \quad b \rightarrow b_{-1}=\frac{c}{V(V-2 \alpha c)}, \\
& X \rightarrow X_{-1}=X-2 \alpha \frac{V c_{x}-V_{x} c}{V(V-2 \alpha c)}, \quad Y \rightarrow Y_{-1}=\frac{V^{2} Y+2 \alpha\left[V V_{x}-c V_{y}-\alpha V\left(c_{x}+c X\right)\right]}{V(V-2 \alpha c)}, \\
& \xi \rightarrow \xi_{-1}=\xi-4 \alpha \frac{\alpha^{2} V\left(c_{x x}+(c X)_{x}\right)-\alpha\left[c\left(V Y_{x}-V_{x y}\right)+V\left(V_{x x}-c_{x} Y\right)\right]-V V_{x} Y}{V(V-2 \alpha c)},
\end{aligned}
$$

where

$$
\begin{aligned}
& M_{1}=\alpha^{3}\left[U^{2} b\left(2 X_{x}-X^{2}\right)+2 U\left(\left(U_{x} b-U b_{x}\right) X+\left(U^{2}\right)_{x} b_{x}+4(U b)^{2} c+\left(U_{x}^{2}-2 U U_{x x}\right) b\right]+\right. \\
& +\alpha^{2} U\left[U\left(U X_{x}+2 b Y_{x}\right)+\left(2 U_{y} b-U U_{x}\right) X-2 U b_{x} Y+4 U^{2} c b-2 U_{x y} b-U U_{x x}+2 b_{x} U_{y}+2 U_{x}^{2}\right]+ \\
& +\alpha\left[U^{3}\left(Y_{x}+X Y+U c\right)+U^{2}\left(b Y^{2}-2 U_{x} Y-U_{x y}\right)+U_{y}\left(\left(U^{2}\right)_{x}-b U_{y}\right)\right]+U^{2} Y\left(U Y-U_{y}\right) \\
& \\
& M_{-1}=\alpha^{3}\left[V^{2} c\left(X^{2}-2 X_{x}\right)-2 V\left(V_{x} c-V c_{x}\right) X-\left(V^{2}\right)_{x} c_{x}-4(V c)^{2} b-\left(V_{x}^{2}-2 V V_{x x}\right) c\right]+ \\
& +\alpha^{2} V\left[V\left(V X_{x}+2 c Y_{x}\right)+\left(2 V_{y} c-V V_{x}\right) X-2 V c_{x} Y+4 V^{2} b c-2 V_{x y} c-V V_{x x}+2 c_{x} V_{y}+2 V_{x}^{2}\right]- \\
& -\alpha\left[V^{3}\left(Y_{x}+X Y+V b\right)+V^{2}\left(c Y^{2}-2 V_{x} Y-V_{x y}\right)+V_{y}\left(\left(V^{2}\right)_{x}-c V_{y}\right)\right]+V^{2} Y\left(V Y-V_{y}\right) .
\end{aligned}
$$

It is possible to verify that $X_{ \pm 1, y}=Y_{ \pm 1, x},\left(b_{1}\right)_{-1}=\left(b_{-1}\right)_{1}=b$ and so on.

To obtain dressing chains of discrete symmetries we must exclude potentials $u, v$, $q$ from the two systems: (47) and and the system of equations obtained from (47) by replacing $a \rightarrow a_{1}, b \rightarrow b_{1}, c \rightarrow c_{1}, d \rightarrow d_{1}, q \rightarrow q_{1}=q+4(a+d)_{x}, u \rightarrow u_{1}, v \rightarrow v_{1}$ (it is necessary to use (45)). As a result, we have y-chains

$$
\begin{aligned}
& {\left[2 b c(d-a)+b_{x} c-b c_{x}\right]\left(a_{1, y}-\alpha a_{1, x}\right)+\left(b c_{1}-b_{1} c\right)\left(a_{y}-\alpha a_{x}\right)_{x}+\left(b c_{1}-b_{1} c\right)\left(b c_{y}-b_{y} c\right)+} \\
& +\alpha\left[\left(b_{1} c+2 b_{1} c_{1}+b c_{1}\right)\left(b c_{x}-b_{x} c\right)+4 b c\left(a c_{1}\left(b+b_{1}\right)-b_{1} d\left(c+c_{1}\right)\right)\right]+ \\
& +\left[\left(b c_{1}+b_{1} c\right)(a-d)+b_{1} c_{x}-b_{x} c_{1}\right]\left(a_{y}-\alpha a_{x}\right)=0 \\
& \left(b c_{1}-b_{1} c\right)\left[\left(b_{1}-b\right)_{y}-\alpha\left(b+b_{1}\right)_{x}\right]+\left(a-a_{1}-d+d_{1}\right)\left[\alpha\left(a_{1, x} b-a_{x} b_{1}\right)+a_{y} b_{1}-a_{1, y} b\right]+ \\
& +2 \alpha\left[\left(b+b_{1}\right)\left(a b c_{1}+c b_{1} d_{1}\right)-b b_{1}\left(c+c_{1}\right)\left(a_{1}+d\right)\right]=0 \\
& \left(b_{1} c-b c_{1}\right)\left[\left(c_{1}-c\right)_{y}+\alpha\left(c+c_{1}\right)_{x}\right]+\left(a-a_{1}-d+d_{1}\right)\left[\alpha\left(a_{1, x} c-a_{x} c_{1}\right)+a_{y} c_{1}-a_{1, y} c\right]- \\
& -2 \alpha\left[\left(c+c_{1}\right)\left(a_{1} c_{1} b+c d b_{1}\right)-c c_{1}\left(b+b_{1}\right)\left(a+d_{1}\right)\right]=0 \\
& \left(a_{1}+d_{1}\right)_{y}-\alpha\left(a_{1}-d_{1}\right)_{x}=0
\end{aligned}
$$


and t-chains:

$$
\begin{aligned}
& \alpha^{2}\left(a_{1}-a\right)_{t}+i\left[2 \alpha ^ { 2 } \left(\left(a^{2}-a_{1}^{2}-2 b_{1} c_{1}-a_{1, x}-d_{x}\right)_{x}+2\left((a+d) b c-\left(b+b_{1}\right) c_{1} d_{1}-\left(c+c_{1}\right) a_{1} b_{1}-\right.\right.\right. \\
& \left.\left.-\left(b c_{1}\right)_{x}\right)-b_{x} c_{1}-b_{1} c_{x}\right)+\alpha\left(\left(c-3 c_{1}\right) U_{x}+\left(b+b_{1}\right) V_{x}+2\left(b_{1} c_{y}-b_{y} c_{1}-(a+d)_{x y}+\right.\right. \\
& \left.\left.\left.+\left(b_{x}+a_{1} b_{1}-a b\right) V-\left(c_{1, x}+c_{1} d_{1}-c d\right) U\right)\right)+\left(b-b_{1}\right) V_{y}+\left(c-c_{1}\right) U_{y}\right]=0 \\
& \alpha^{2}\left(b b_{1, t}-b_{t} b_{1}\right)+i\left[2 \alpha ^ { 2 } \left(2 b^{2}\left(a_{1} d_{1}-b_{1} c_{1}\right)-2 b b_{1}^{2}\left(c_{1}+c\right)+b_{x x} b_{1}-b_{1, x x} b-2 b d_{1, x}\left(b+b_{1}\right)+\right.\right. \\
& \left.+2\left(b_{x} d b_{1}-b_{1, x} d_{1} b\right)+b\left[a_{1} b_{x}-2 a_{1, x} b_{1}-b_{x x}-3 b_{x} d_{1}+2\left(b_{1}\left(d^{2}-b c\right)-d_{1}^{2}\left(b+b_{1}\right)\right)\right]\right)+ \\
& +\alpha\left(\left(b_{1}-b\right) U_{x x}+\left(3\left(b_{1} d-b d_{1}\right)+a_{1} b-a b_{1}\right) U_{x}+2\left[b_{1} b V\left(b+b_{1}\right)+b b_{y}\left(a_{1}-d_{1}\right)-b b_{x y}+\right.\right. \\
& \left.\left.+U\left(b_{1} d_{x}-b d_{1, x}+b_{1} d^{2}-b d_{1}^{2}-b b_{1}\left(c+c_{1}\right)+b a_{1} d_{1}-b_{1} a d\right)\right]\right)+U_{x y}\left(b_{1}-b\right)+ \\
& \left.+U_{y}\left(b\left(a_{1}-d_{1}\right)-b_{1}(a-d)\right)\right]=0 \\
& \alpha^{2}\left(c c_{1, t}-c_{t} c_{1}\right)+i\left[2 \alpha ^ { 2 } \left(2 c^{2}\left(b_{1} c_{1}-a_{1} d_{1}\right)+2 c c_{1}^{2}\left(b_{1}+b\right)+c_{1, x x} c-c_{1} c_{x x}+2 c a_{1, x}\left(c+c_{1}\right)+\right.\right. \\
& \left.+2\left(c a_{1} c_{1, x}-c_{1} a c_{x}\right)+c\left[2 c_{1} d_{1, x}-d_{1} c_{x}+c_{x x}+3 c_{x} a_{1}+2\left(c_{1}\left(b c-a^{2}\right)+a_{1}^{2}\left(c+c_{1}\right)\right)\right]\right)+ \\
& +\alpha\left(\left(c_{1}-c\right) V_{x x}+\left(3\left(a c_{1}-a_{1} c\right)+c d_{1}-c_{1} d\right) V_{x}+2\left[c_{1} c U\left(c+c_{1}\right)+c c_{y}\left(d_{1}-a_{1}\right)-c c_{x y}+\right.\right. \\
& \left.\left.+V\left(c_{1} a_{x}-c a_{1, x}+c_{1} a^{2}-c a_{1}^{2}-c c_{1}\left(b+b_{1}\right)+c a_{1} d_{1}-c_{1} a d\right)\right]\right)+V_{x y}\left(c-c_{1}\right)+ \\
& \left.+V_{y}\left(c\left(a_{1}-d_{1}\right)-c_{1}(a-d)\right)\right]=0, \\
& \alpha^{2}\left(d_{1}-d\right)_{t}+i\left[2 \alpha^{2}\left(\left(d_{1}^{2}-d^{2}+2 b_{1} c_{1}+a_{x}+d_{1, x}\right)\right)_{x}-2\left((a+d) b c-\left(b+b_{1}\right) c_{1} d_{1}-\left(c+c_{1}\right) a_{1} b_{1}-\right.\right. \\
& \left.\left.-\left(b_{1} c\right)_{x}\right)+b_{x} c_{1}+b_{1} c_{x}\right)+\alpha\left(\left(b-3 b_{1}\right) V_{x}+\left(c+c_{1}\right) U_{x}+2\left(c_{1} b_{y}-b_{1} c_{y}-(a+d)_{x y}+\right.\right. \\
& \left.\left.\left.+\left(c_{x}+c_{1} d_{1}-c d\right) U-\left(b_{1, x}+a_{1} b_{1}-a b\right) V\right)\right)+\left(c_{1}-c\right) U_{y}+\left(b_{1}-b\right) V_{y}\right]=0 .
\end{aligned}
$$

These formulas have awful sight! Unfortunately, I don't know how to write them in more compact form in our gauge. (51) has the best form which I could imagine.

Although this equations are long and difficult, the end result is quite simple. We can see that all of $(1+1)$ NLS formalism carried over directly into the $(2+1)$ DS dressing chains. In particular, the chains (51) involve $L A$ pair for the $\operatorname{mDS}(49)$. We do not give it here.

As noted in Sec. 3, the KP equations admit two types of chains, which we call conjugate. It is a new result, which is characteristic precisely of multidimensional systems. Of course, it is truth for the DS equations.

To construct the chains which are conjugate to (50)-(51), we must introduce the new $L A$ pair for the DS equations $(p=p(x, y, t), f=f(x, y, t))$ :

$$
\begin{aligned}
& p_{y}=\alpha p_{x}-v f, \quad f_{y}=-\alpha f_{x}-u p \\
& p_{t}=-2 i p_{x x}+\frac{2 i}{\alpha} v f_{x}-\left(\frac{1}{2}\left[\frac{1}{\alpha} F_{y}+F_{x}\right]-\frac{i}{\alpha^{2}} u v\right) p+\frac{i}{\alpha^{2}}\left(\alpha v_{x}+v_{y}\right) f, \\
& f_{t}=2 i f_{x x}+\frac{2 i}{\alpha} u p_{x}-\left(\frac{1}{2}\left[\frac{1}{\alpha} F_{y}-F_{x}\right]+\frac{i}{\alpha^{2}} u v\right) f+\frac{i}{\alpha^{2}}\left(\alpha u_{x}-u_{y}\right) p
\end{aligned}
$$

two matrix functions

$$
\Psi=\left(\begin{array}{cc}
\psi_{1} & \psi_{2} \\
\phi_{1} & \phi_{2}
\end{array}\right), \quad \Phi=\left(\begin{array}{ll}
p_{1} & f_{1} \\
p_{2} & f_{2}
\end{array}\right)
$$

and one-form

$$
d \Omega=\Phi \Psi d x+\alpha \Phi \sigma_{3} \Psi d y+2 i\left(\Phi \sigma_{3} \Psi_{x}-\Phi_{x} \sigma_{3} \Psi+\frac{1}{\alpha} \Phi \sigma_{3} \Psi\right), \quad \Omega=\int d \Omega .
$$


This one -form is closed if $\psi_{1,2}, \phi_{1,2}, p_{1,2}$ and $f_{1,2}$ are solutions of Eqs. (44) and (52) for the same $u, v F$. It is easy to see that the quantities

$$
\begin{array}{ll}
A=\frac{p_{1, x} f_{2}-p_{2, x} f_{1}}{\Delta}, & B=\frac{f_{1, x} f_{2}-f_{2, x} f_{1}}{\Delta}, \quad C=\frac{p_{2, x} p_{1}-p_{1, x} p_{2}}{\Delta}, \\
D=\frac{f_{2, x} p_{1}-f_{1, x} p_{2}}{\Delta}, & \Delta=p_{1} f_{2}-p_{2} f_{1} .
\end{array}
$$

are solutions of the $y$-system

$$
\begin{aligned}
& A_{y}=\alpha\left(A_{x}+2 B C\right)+u C-v B, \quad B_{y}=-\alpha\left(B_{x}+2 A B\right)+(D-A) u-u_{x} \\
& C_{y}=\alpha\left(C_{x}+2 C D\right)+(A-D) v-v_{x}, \quad D_{y}=-A_{y}+\alpha(A-D)_{x} .
\end{aligned}
$$

Here, we don't need $t$-system (four equations for the $A_{t}, B_{t}, C_{t}$ and $D_{t}$ ).

The Darboux transformation (45) (for the system (44)) give us the transformation rule for the functions $A, B, C D$

$A \rightarrow a_{1}=\Lambda_{11}-a, \quad B \rightarrow b_{1}=\Lambda_{12}-b, \quad C \rightarrow c_{1}=\Lambda_{21}-c, \quad D \rightarrow d_{1}=\Lambda_{22}-d$,

where $a, b, c$ and $d$ are defined in (46), and $\Lambda_{i k}$ are elements of the matrix $\Lambda=\Psi \Omega^{-1} \Phi$, $i, k=1,2$. Transforming the system (53) and exluding potentials $u$ and $v$ from this system and from the first four equations (47) we get $y$-chains which are conjugate to (50). There are four equations, two of them are equaivalent to the first and fourth equations (50). The rest two equations are given by

$$
\begin{aligned}
& \left(b c_{1}-b_{1} c\right)\left[\left(b+b_{1}\right)_{y}+\alpha\left(b+b_{1}\right)_{x}\right]+\left(a+a_{1}-d-d_{1}\right)\left[\alpha\left(a_{x} b_{1}-a_{1, x} b\right)+a_{1, y} b-a_{y} b_{1}\right]- \\
& -2 \alpha\left[\left(b+b_{1}\right)\left(a b c_{1}+a_{1} b_{1} c\right)-b b_{1}\left(c+c_{1}\right)\left(d+d_{1}\right)\right]=0 \\
& \left(b_{1} c-b c_{1}\right)\left[\left(c+c_{1}\right)_{y}-\alpha\left(c+c_{1}\right)_{x}\right]+\left(a+a_{1}-d-d_{1}\right)\left[\alpha\left(a_{x} c_{1}-a_{1, x} c\right)+a_{1, y} c-a_{y} c_{1}\right]+ \\
& +2 \alpha\left[\left(c+c_{1}\right)\left(b c_{1} d_{1}+b_{1} c d\right)-c c_{1}\left(b+b_{1}\right)\left(a+a_{1}\right)\right]=0 .
\end{aligned}
$$

The conjugate t-chains can be obtained by the same way. We do not give them here.

\section{On the localized solutions of the DS equations}

Discrete symmetries are a good way to obtain exact solutions of the nonlinear integrable equations. In [14] we applied the DT (S-symmetry) to construct exact solutions to the DS-I and DS-II equations (the same to the BLP equations see in [15]). In particular, we have obtained the dromion solutions of the DS-I equations. In this section we use the T-symmetry to construct nonsingular solutions to the DS-I that fall off according to the exponential and/or rational low along all directions in the plan. In addition to the known dromion, we give several new solutions with above properties. In rest path of this section we present (via S-symmetrie) novel exact solution of the DS-II equations describing the soliton on the plane wave background.

1. DS-I equations. 
To study the DS-I equations it is convenient to use the following change of variables:

$$
\partial_{x} \rightarrow \frac{1}{\sqrt{2}}\left(\partial_{x}+\partial_{y}\right), \quad \partial_{y} \rightarrow \frac{1}{\sqrt{2}}\left(\partial_{x}-\partial_{y}\right), \quad v \rightarrow-\frac{\bar{u}}{2} .
$$

We are interested of localized solutions to the DS-I equations which move without shape distortions and we look for these solutions in the form

$$
u(t, x, y)=\overline{v(t, x, y)}=U(\xi, \eta) \exp (i \theta), \quad q(t, x, y)=Q(\xi, \eta)
$$

where

$$
\xi=x-2 a t, \quad \eta=x-4 b t, \quad \theta=a x+b y-\left(a^{2}+b^{2}\right) t,
$$

and $U(\xi, \eta)$ is the real function. It was shown in [16] that a solutions to the DS-I equations can be found in this setting from the nonlinear Liouville equation

$$
\partial_{x} \partial_{y} \Phi=\frac{1}{2} \exp (\Phi)
$$

for the function $\Phi \equiv \log \left(U^{2}\right)$. This result was obtained via the T-symmetries (see the previous section).

It is convenient to introduce the boundary conditions for the $U(x, y)$ as

$$
U(x, 0)=A(x), \quad U(0, y)=B(y), \quad A(0)=B(0)=C,
$$

where $A(x)$ and $B(y)$ are the given functions. Now we can use the solution to the Gousat problem for (54) given in [17], from which we find

$$
U(x, y)=\frac{4 C A(x) B(y)}{4 C^{2}-\int_{0}^{x} d p A^{2}(p) \int_{0}^{y} d q B^{2}(q)} .
$$

Using T-symmetries, one can also find the explisit expression for the $Q(x, y)$ field

$$
\begin{aligned}
& Q(x, y)=\frac{1}{2}\left(P^{\prime}\left(\frac{1}{P^{\prime}}\right)^{\prime \prime}+G^{\prime}\left(\frac{1}{G^{\prime}}\right)^{\prime \prime}\right)-\frac{3}{4}\left(\left[\left(\log P^{\prime}\right)^{\prime}\right]^{2}+\left[\left(\log G^{\prime}\right)^{\prime}\right]^{2}\right)+ \\
& +\frac{2}{P G-4}\left(P G^{\prime \prime}+P^{\prime \prime} G-\frac{\left(P G^{\prime}\right)^{2}+\left(P^{\prime} G\right)^{2}+8 P^{\prime} G^{\prime}}{P G-4}\right),
\end{aligned}
$$

where

$$
P(x)=\frac{1}{C} \int_{0}^{x} d p A^{2}(p), \quad G(y)=\frac{1}{C} \int_{0}^{y} d q B^{2}(q) .
$$

Expression (56) also allows us to construct localized solutions. For example, choosing the boundary functions in the form of two solutions of the NLS equation,

$$
A(x)=\frac{a}{\cosh (\alpha x)}, \quad B(y)=\frac{a}{\cosh (\beta y)},
$$

we obtain the well-known "one-dromion" solution [18]. Other localized objects are found by choosing the boundary conditions appropriately. Thus, assuming $A(x)$ and $B(y)$ to be solutions of the KdV equation, we obtain a new solution that decays exponentially in all directions,

$U(x, y)=\frac{4 a \alpha \beta}{(\cosh (\alpha x) \cosh (\beta y))^{2}\left[4 \alpha \beta-a^{2} \tanh (\alpha x) \tanh (\beta y)\left(1-\frac{\tanh ^{2}(\alpha x)}{3}\right)\left(1-\frac{\tanh ^{2}(\beta y)}{3}\right)\right]}$, 
where $a, \alpha$ and $\beta$ are real constants satisfying the condition $a^{2}<9 \alpha \beta$, which guarantees the nonsingular behaviour of (58). Let us note that if if the functions $A(x)$ and $B(y)$ have maxima $m$ and $l$, respectively, the solution $U(x, y)$ determined by Eq. (56) corresponds to $m l$ localized bound formations. It possible to show that these equations are different from the (L,M)-dromions [19] and $N^{2}$-soliton solutions built in [20] and [21].

The rational localized solutions ("lumps") are obtained in the same way. let us consider, for example, the following boundary conditions:

$$
\begin{array}{ll}
A_{1}(x)=\frac{\alpha_{1}}{x^{2}+a^{2}}, & A_{2}(x)=\frac{\alpha_{2}}{x^{4}-(a x)^{2}+a^{4}}, \\
B_{1}(y)=\frac{\beta_{1}}{y^{2}+b^{2}}, & B_{2}(y)=\frac{\beta_{2}}{y^{4}-(b y)^{2}+b^{4}} .
\end{array}
$$

Substituting $A_{1}$ and $B_{1}$ into (56) and using (55), one obtains the one-lump solution,

$$
\begin{gathered}
u=-16 C a^{2} b^{2} /\left(C^{2} b^{2} a^{2} y x-16\left(x^{2}+a^{2}\right)\left(y^{2}+b^{2}\right)\right. \\
\left.+a b C^{2}\left(S Q\left(x^{2}+a^{2}\right)\left(y^{2}+b^{2}\right)+y b S\left(x^{2}+a^{2}\right)+x a Q\left(y^{2}+b^{2}\right)\right)\right), \\
S=\arctan \left(\frac{x}{a}\right), \quad Q=\arctan \left(\frac{y}{b}\right) .
\end{gathered}
$$

Substituting $A_{1}$ and $B_{2}$ (or $A_{2}$ and $B_{1}$ ), we get the two-lamp solution, and substituting $A_{2}$ and $B_{2}$, the four-lamp solution. One can always choose the paramerers involved in (59) such that the solutions falling off in all directions according to rational law are nonsingular. Finally, choosen $A(x)$ from (57) and $B_{1}(y)$ from (59), we obtain the localized solution falling off according to the exponential law as a function of $x$ and according to the ratianal law as a function of $x$

\section{DS-II equations.}

We couldn't find a localized nonstationary solution of the DS-II equation. We present only soliton on the plane wave background. We choose the initial solution of the DS-II equations as

$$
u=A \exp (i S), \quad S=-\left(2 A^{2}+a^{2}-b^{2}\right) t+a x+b y, \quad q=0 .
$$

where $A, a$ and $b$ are real constants. The solutions of the $L A$ pair (44)

$$
\psi_{1}=f \exp \left(i \frac{S}{2}+M\right), \quad \psi_{2}=\overline{\frac{i(2 m-b)-p}{2 A}\left(f-2 i \frac{\alpha_{1}}{p}\right)} \exp \left(i \frac{S}{2}+\bar{M}\right),
$$

where

$$
\begin{aligned}
& f=\alpha_{1}\left[x+\frac{1}{p}\left((b-2 m) y+2\left(2\left(b m-A^{2}-2 m^{2}\right)-a p\right) t\right)\right]+\alpha_{2}, \\
& M=m x+\frac{1}{2}((p-a) y+[p(b+2 m)-4 a m] t), \quad p^{2}+\left[4 A^{2}+(b-2 m)^{2}\right]^{2}=0 .
\end{aligned}
$$

Using (45) (with $\bar{m}=m, \overline{\alpha_{1}}=\alpha_{1}$ and $\alpha_{2}=0$ ) we get nonsingular solution $u_{1}$ which is the soliton on the plane wave background:

$$
\left|u_{1}\right|^{2} \rightarrow A^{2} \quad \text { at } \quad x^{2}+y^{2} \rightarrow \infty
$$

This solution is two-dimensional generalization of "exulton" solution of the NLS built in [1]. 


\section{Conclision}

The past years of intense theoretical research have made it increasingly clear that the secret to integrability most likely lies in the power of discrete symmetries. Let us summarize some of the promising features of the discrete symmetries:

(1) It is the an extremely power method to construct exact solutions of integrable equations.

(2) This approach includes all "soliton miracles": finit-gap solutions, the Painleve property ets.

(3) Discrete symmetries allow us to proliferate integrable equations. For example, the whole MKdV theory can be consider as the DT theory and the Toda-Volterra theory can be consider as the theory of S- and T-symmetries of the NLS equations [9].

(4) Discrete symmetries led to connection between integrable system and supersymmetry.

(5) Discrete symmetries allow us to construct discrete integrable systems.

Ideally, we would want a unified theory to unite and understand all "soliton miracles" . There are two ways to find this unified theory. The first path is connected with the Hirota bilinear difference equation [22]. This famous equation is known to provide a canonical integrable discretization for most important types of soliton equation.

The second path is the theory of dressing chains. This approach allow us to proliferate integrable equations and, at the same time, to establish a links between known integrable equations (it is clear that the Miura map can be obtained from the dressing chains). Ideally, we want to show that all integrable equations are nothing but different forms of a single equation!

Even if this direction is right, it is still a long way off. Now the theory itself often seems like a confused jumble of random (but usefull) rules and random (but remarkable) observations. It remains to be seen how useful the dressing chains will become in the future.

\section{Acknowledgement}

This work was supported by RFBR, Grant 00-01-00783 and the Grant of Education Department of the Russian Federation, No. E00-3.1-383.

\section{References}

1. V.B. Matveev and M.A. Salle, Darboux Transformations and Solitons, Springer, Berlin (1991).

2. B.A. Borisov and S.A.Zykov, Theor. Math. Phys., 115, 530 (1998).

3. A.V. Mikhailov, A.B. Shabat and R.I. Yamilov, Russ. Math. Surv., 42, 1 (1987).

4. A.V. Yurov, Theor. Math. Phys., 119, 731 (1999).

5. V.A. Andreev and M.V. Burova, Theor. Math. Phys., 85, 376 (1990).

6. V.G. Konopelchenko and V.G. Dubrovsky, Stud. Appl. Math., 86, No.3, 219 (1992). 
7. A.P. Veselov and A.B. Shabat, Funct. Anal. Appl., 27, 10 (1993).

8. A.B. Shabat, Theor. Math. Phys., 121, 165 (1999).

9. .B. Shabat and R.I. Yamilov, Algebra and Analis., 2, 183 (1990).

10. A. Davey and K. Stewartson, Proc. R. Soc. A, 338, 101 (1974).

11. K. Nishinari, K. Abe and J. Satsuma, Theor. Math. Phys., 99, 745 (1994).

12. A.V. Yurov, Theor. Math. Phys., 109, 1508 (1996).

13. A.N. Leznov, A.B. Shabat, R.I. Yamilov., Phys. Lett. A., 174, 397 (1993).

14. S.B.Leble, M.A. Salle and A.V. Yurov, Inverse Problems, 8, 207 (1992).

15. A.V. Yurov, Phys. Lett. A., 262, 445 (1999).

16. A.V. Yurov, Theor. Math. Phys., 112, 1113 (1997).

17. G.P. Dzhordzhadze (Jorjadze), Theor. Math. Phys., 41, 867 (1979).

18. M. Boiti, J.J.-P. Leon, L. Martina and F. Pempinelli, Phys. Lett. A, 132, 432 (1988).

19. A.S. Fokas and P.M. Santini, Phys. Rev. Lett., 63, 1329 (1989).

20. M. Boiti, L. Martina, O.K. Pashaev and F. Pempinelli, "Dinamics of Multidimensional Solitons", Lecce preprint (June 1991).

21. J. Hietarinta and R. Hirota, Phys. Lett. A, 237, 145 (1990).

22. R. Hirota, J. Phys. Soc. Japan, 50, 3785 (1981). 